\title{
Oral Mini Pulse Therapy a Novel Therapy in a Management of Multivariant Oral Lichen Planus: An Unusual Case Report
}

\author{
Aditya Dupare $^{1 *}$, Apeksha S Dhole ${ }^{2}$ and Mukta Motwani \\ ${ }^{1}$ Department of Oral Medicine and Radiology, Yogita Dental College and Hospital, Khed, Maharashtra, India \\ ${ }^{2}$ Department of Oral Medicine and Radiology, V.S.P.M. Dental College, Nagpur, Maharashtra
}

\begin{abstract}
Oral lichen planus (OLP) is a chronic mucocutaneous disease with an unknown aetiology, affecting $0.5 \%-2 \%$ of the population and with a predilection for females in fourth to fifth decade of life. Most oral lichen planus lesions are asymptomatic but the atrophic and erosive forms of OLP can cause symptoms ranging from spontaneous soreness to severe pain interfering with eating, speech and swallowing. Various drugs have been used for the treatment of OLP including corticosteroids and other immunomodulators. However, no therapy is considered as the single most effective and without side effects in the management of this enigmatic disease. This is a case report of successful management of extensive, symptomatic multivariant OLP with a novel treatment with oral mini pulse (OMP) therapy.
\end{abstract}

Keywords: Oral lichen planus (OLP); Betamethasone; Oral mini pulse (OMP) therapy

\section{Introduction}

In 1869 Erasmus Wilson described Lichen planus as a pruritic dermatosis of unknown etiology that affect the skin and mucous membrane [1]. In the oral cavity it involves the buccal mucosa, tongue and gingiva, floor of mouth lips and palate. Usually, OLP has distinctive clinical features and distribution, characterized by multiple, bilateral but not always symmetrical, mucosal lesions. There are four clinical manifestations of OLP: popular, reticular, erythematous (atrophic) and erosive (ulcerated, bullous) [2].

Carrozzo and Gandolfo described topical and systemic corticosteroid management protocol. Topical corticosteroids are used as the $1^{\text {st }}$ line drugs, particularly for mild lesions, and systemic corticosteroids indicated for multiple, widespread and unresponsive lesions [3]. The reported side effects associated with corticosteroids are secondary infections, osteoporosis, diabetes, psychological and cardiovascular disorders. Hence, there has been a considerable effort to find alternative methods of treatment [4].

In the past few years, pulse therapy (PT) has been widely used in the treatment of various immunological and dermatological disorders. It was first described by Pasricha and Ramji in 1984. It is defined as discontinuous or intermittent intravenous infusion of very high doses of drugs over a short time. The aims of PT's are to achieve more rapid and effective disease control compared with conventional oral dosing, thus allowing a reduction in a long-term maintenance corticosteroids doses and their side effects. However, the patients on corticosteroids PT have to be continuously monitored in a set up because high dose of drugs are given. This may be particularly unnecessary in patients with only oral lesions without dermatological involvement. To overcome this problem, around 20 years ago a new therapeutic regimen called oral mini pulse therapy (OMP) were introduced allowing for oral administration of drugs with lesser dosages $[5,6]$.

The OMP therapy was primarily designed for treating patients with vitiligo to achieve the therapeutic results with minimum side effects [7]. In present case report, a symptomatic multivariant of OLP successfully treated with novel OMP therapy.

\section{Case Report}

A 20-year female girl from Balaghat MP visited the department of
Oral diagnosis, medicine and radiology, V.S.P. M's dental college and research Centre, Nagpur, Maharashtra, India with a chief complaint difficulty in mastication due to burning sensation and ulceration in the oral cavity for 2 months. The burning sensation was gradual in onset and aggravates on consumption of hot and spicy foodstuffs. She was visited private medical practitioner in Balaghat where she advised the medication for Clenorush gel (Clotrimazole 1\%) for 1 month.

Simultaneously, she started taking ayurvedic medication prescribed by another practitioner for the same for about $1 / 2$ months. Since she did not get relieved, so she discontinued the all the medication by her own. She had negative history of itching or burning over the other parts of body and similar lesion over the other part of body. Her pervious medical, family and personal history was non-significant. She had dental history with endodontic treatment in lower left back region, and history of extraction and restoration in lower right back region approx. 2 years back. She had a habit of chewing kharra and sweet supari since 3-years, 4-5 time a day but now she had quit the habit 2 months back. On general physical examination no significant abnormality was seen. Vital signs were within the normal limits. On intra oral soft tissue examination, on left and right buccal mucosa, grayish white nonscrapable keratotic patch seen on left and right buccal mucosa showing reticular pattern with surrounding atrophic areas. Lesion showed characteristics Wickham striate features suggestive of atrophic and reticular type of lichen planus (Figure 1). On dorsal surface of tongue, plaque like whitish keratotic patches seen on middle $1 / 3^{\text {rd }}$ of tongue. Size of patch approx. $3 \mathrm{~cm} \times 1 \mathrm{~cm}$. Also, similar small keratotic patch seen on the tip of the tongue (Figure 2). Ventral surface of tongue appears normal. On palate, Multiple small fluid containing vesicles with atrophic areas seen. Sizes of blisters ranging from 0.5 to $1 \mathrm{~mm}$ in diameter, these features suggestive of bullous type of lichen planus

${ }^{*}$ Corresponding author: Aditya Dupare, Department of Oral Medicine and Radiology, Yogita Dental College and Hospital, Khed, Maharashtra India, Tel: +91 9665559075; E-mail: adupare@gmail.com

Received: March 03, 2018; Accepted: March 28, 2018; Published: March 31, 2018

Citation: Dupare A, Dhole AS, Motwani M (2018) Oral Mini Pulse Therapy a Novel Therapy in a Management of Multivariant Oral Lichen Planus: An Unusual Case Report. Oral health case Rep 4: 145. doi:10.4172/2471-8726.1000145

Copyright: $\odot 2018$ Dupare A, et al. This is an open-access article distributed under the terms of the Creative Commons Attribution License, which permits unrestricted use, distribution, and reproduction in any medium, provided the original author and source are credited. 
(Figure 3). On labial and palatal aspect of maxillary gingiva shows a red and inflamed areas of desquamation were seen. Visible as well as discharge on provocation also present (Figure 4). On complete clinical case analysis, provisional diagnosis of multivariant OLP along with chronic desquamative gingivitis (Gingivosis) were given. Following patient informed concern, patients routine complete blood count and punch biopsy were performed of left buccal mucosa and specimen were transferred to histopathological examination. Histopathological examination showed stratified squamous epithelium with hyperorthokeratosis. There was liquefaction degeneration of basal cell layer, sub epithelial dense band like infiltration of chronic inflammatory cells. Connective tissue stroma showed fibro cellular along with of muscle tissue given the confirmatory diagnosis of OLP (Figure 5).
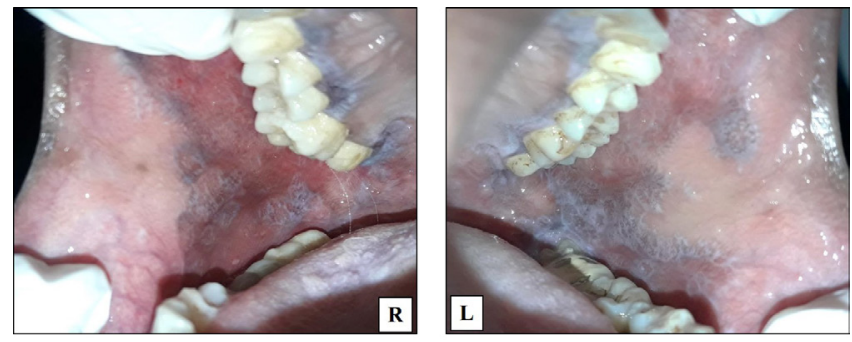

Figure 1: Reticular and erosive oral lichen planus seen on the right and left buccal mucosa.

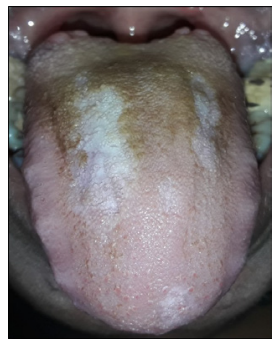

Figure 2: Plaque type of oral lichen planus on the dorsum of tongue.

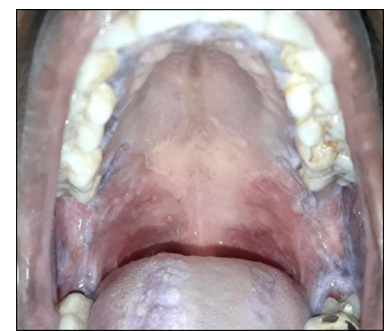

Figure 3: Bullous type of lichen planus on the hard and soft palate.

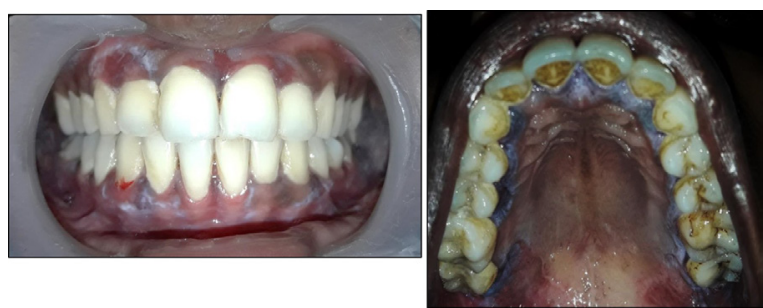

Figure 4: Desquamation of gingiva on the labial and aspect of maxilla and mandible and palatal aspect of maxillary arch only.

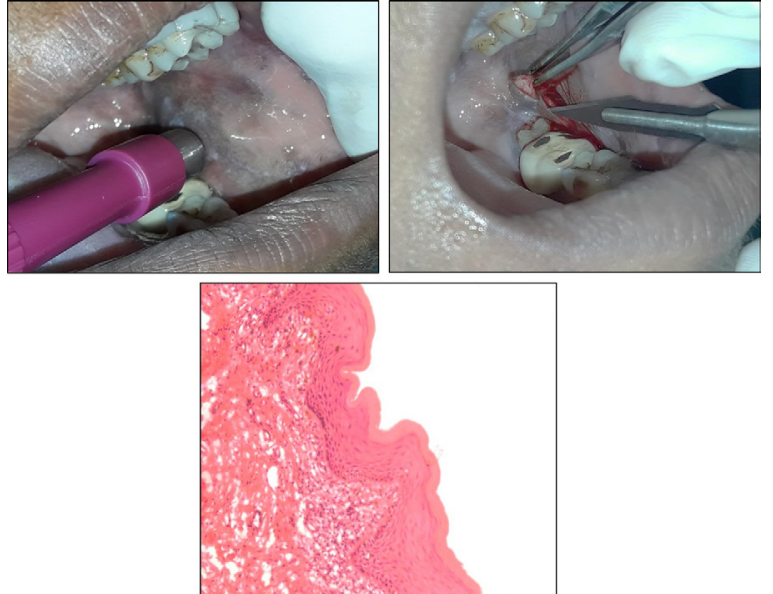

Figure 5: Punch biopsy along with histopathology $\mathrm{H}$ \& E section representing oral lichen planus.
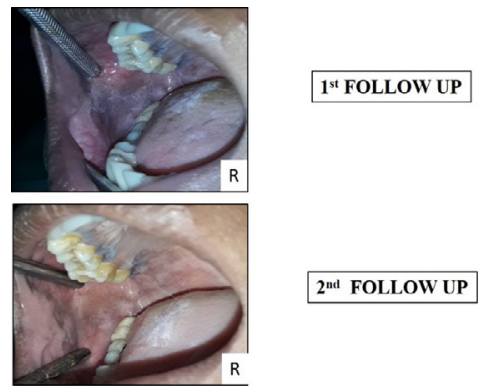

$2^{\text {nd }}$ FOLLOW UP


Figure 6: 3 steps follow up results on right and left buccal mucosa.

The patients counselling was done regarding maintenance of oral hygiene, diet and rising with benzyldiazine hydrochloride mouth rinse twice a day for 1 month. The regimen was started with Betamethasone $(10 \mathrm{mg})$ in two divided doses/Week for 1 month (Friday: $5 \mathrm{mg}$ and Saturday: $5 \mathrm{mg} /$ Day). The ulcerative lesions and erythema and burning were reduced up to $50 \%$ but still few persistent vesicles were seen of palate. Desquamation were also reduced to $75 \%$. Lesion over the tongue also get reduced significantly. On $1^{\text {st }}$ recall visit dose was reduced to $5 \mathrm{mg}$ in two divided doses/Week for 1 month (Friday: $2.5 \mathrm{mg}$ and Saturday: $2.5 \mathrm{mg} /$ Day). After $1^{\text {st }}$ recall, patient reported with complete reduction of burning sensation desquamation and vesicular eruption. Tongue lesion reduced but still persist on dorsum surface of tongue. On $2^{\text {nd }}$ visit recall complete hemogram were done. Hemogram report were within normal limit. Then, dose of $5 \mathrm{mg}$ was maintained for more 20 days (Friday: $2.5 \mathrm{mg}$ and Saturday: $2.5 \mathrm{mg}$ /Day) then withdrawal of drug was done. During each visit patient was monitored at regular intervals where weight gain and hematological investigation within normal limits. On post therapy follow-up 2 month no new lesion was seen. Diffuse white asymptomatic patch persists over the dorsum of tongue (Figures 6 and 7).

\section{Discussion}

Oral lichen planus is a chronic inflammatory condition that 


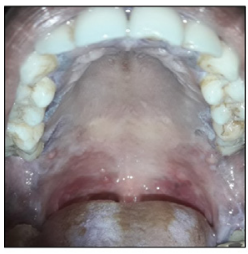

$1^{\text {st }}$ FOLLOW UP

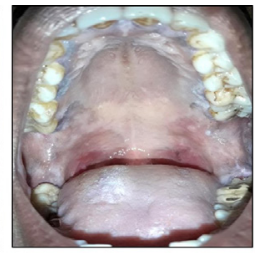

$2^{\text {nd }}$ FOLLOW UP

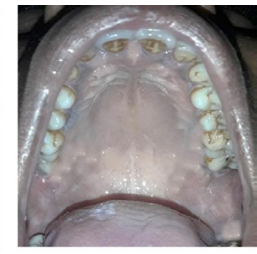

$3^{\text {rd }}$ FOLLOW UP
Figure 7: 3 steps follow up results of hard and soft palate.

is probably of multifactorial origin, often idiopathic with immune pathogenesis involving T-cells. The disease affects $0.5-2 \%$ of population with a female's predilection and the mean age of onset in the $4^{\text {th }}-5^{\text {th }}$ decade of life. Its chronic remitting and relapsing course with spontaneous remission making management of OLP a challenging for clinicians $[3,8]$.

The most widely accepted treatment for lesions of OLP involves topical or systemic corticosteroids to modulate immune response of patient [9]. The corticosteroids are most effective agents in the treatment of OLP, with topical agents being preferred over systemic drugs except during acute conditions. It is also uncomfortable to the patient to use topical medication frequently. Systemic corticosteroids, are useful during the acute conditions and are often used in combination with topical steroids. The long-term side effects of systemic steroids are also common. In OMP therapy, corticosteroids have shown better efficacy with few and acceptable adverse effects, in diseases like vitiligo and alopecia areata [10]. OMP was used for the administration of betamethasone ( $5 \mathrm{mg}$ ) orally once daily for two consecutive days in a week for a period of three weeks to three months. A many literature reviews a very few case reports and series where OMP has been used effectively with minimum side effects to treat OLP.

Mittal et al. in 2000 conducted a study in 10 patients of OLP, between age range of 7-60 years, who had experienced OLP for a period of one month to two years. The patients were managed with $5 \mathrm{mg}$ oral betamethasone on two consecutive days in a week for 3 months. They reported with an excellent response in $60 \%$ patients and a good $50 \%-75 \%$ response in $40 \%$ patients. Also, there was no significant side effects noticed from the OMP therapy. Hence, they concluded that OMP therapy may be a safe, effective therapeutic alternative for the management of OLP [11].

Malhotra et al. in 2008 performed a study on 49 patients of OLP were randomly received either OMP comprising oral betamethasone $(5 \mathrm{mg})$ on two consecutive days per week in group A or triamcinolone acetonide $(0.1 \%)$ paste application thrice a day in group B for 3 months followed by tapering during the next 3 months. Treatment response was assessed and changes in the symptoms and side effects were also recorded. A good to excellent response was reported in $68.0 \%$ patients in group A as compared with $66.0 \%$ in group B at 6 months. Asymptomatic state was achieved in 52\% patients in group A and $50 \%$ in group B. Relapse occurred in 9 of $39.1 \%$ patients in group A. So, they concluded that OMP convenient alternative monotherapy to achieve rapid relief in exacerbating state of OLP [10].

Similarly, in 1999 Joshi et al treated a patient with generalized and bullous OLP with OMP therapy consisting of betamethasone $(5 \mathrm{mg}$ ) as a single daily dose on two consecutive days every week. Also, for oral and genital lesions betamethasone dipropionate $(0.01 \%$ gel) twice daily for topical application was advised. OMP was tapered it by 0.5 mg every week and was completely stopped in next 10 weeks. No side effects as well as relapse were reported during the follow-up period of 12-month [12].

In the year 2008, Rashid et al. conducted similar study on 40 patients with OLP, amongst which 20 patients received betamethasone ( $5 \mathrm{mg}$ ) with loratadine for two consecutive days in a week for 6 weeks and 20 patients in group $B$ received a loratadine tablet daily for the span. It was observed that OLP lesions were significantly decreasing in group A. So, they concluded that OMP therapy was found to be more effective than loratadine in the management of OLP [13].

The similar study results were synchronously reported in our case also. In our case, a clear dosage schedule was prescribed to the patient, so that the patient took the medication on the particular day only. Previous studies also they had used betamethasone in OMP and the same regimen was followed in our case patient also. However, other corticosteroids such as methyl prednisolone and dexamethasone also be tried as OMP. In some dermatological lesions, they have achieved better results as compared to betamethasone and hence future studies should be conducted to compare the efficacy of different corticosteroids in the management of OLP.

In the present case, major adverse effects were easily avoided without compromising the clinical outcome. The Major benefit of OMP is its easy administration without hospital admission, unlike PT.

\section{Conclusion}

In conclusion, the present case provides that OMP therapy is a better treatment modality to manage multivariant OLP lesions. The advantages of OMP are its convenient dosage schedule, efficacy, minimal side effects and no suppression cortisol production. Hence, OMP therapy can be employed as a routine treatment modality for OLP as it offers a non-invasive mode that yields significant improvements in the clinical condition of patient.

\section{References}

1. Oztat $\mathrm{P}$, Onder M, Ylter N, Oztat MO (2003) Childhood lichen planus with nail involvement: a case. Turk J Pediatr 45: 251-253.

2. Carrozzo M, Thorpe RJ (2009) Update on oral lichen planus. Expert Rev Dermatol 4: 483-494.

3. Kumar P, Bahirwani S, Raja JV, Pujari M, Tuteja M, et al. (2013) Oral mini pulse therapy: Report of a case and review of the literature. Oral Health Dent Manag 12: 112-118.

4. Rosenberg FR, Sanders S, Nelson CT (1976) Pemphigus: a 20-year review of 107 patients treated with corticosteroids. Arch Dermatol 112: 962-970.

5. Raviraj J, Nayak AG (2007) Current pathophysiological aspects and therapeutic modalities for pemphigus vulgaris: A review. J Indian Acad Oral Med Radiol 19: 503-511.

6. Pasricha JS, Khaitan BK (1993) Oral mini pulse therapy with betamethasone in vitiligo patients having extensive or fast spreading disease. Int J Dermatol 32: 753-757.

7. Waller DG, Renwick AG, Hillier K (2001) Corticosteroids glucocortisteroids and mineralocorticosteroids in: Medical Pharmacology and Therapeutics. Edinburgh: WB Saunders, UK.

8. Thongprasom K, Dhanuthai $\mathrm{K}(2008)$ Steriods in the treatment of lichen planus: a review. J Oral Sci 50: 377-385.

9. Edwards PC, Kelsch R (2002) Oral lichen planus: Clinical presentation and management. Can Dent Assoc 68: 494-499.

10. Malhotra AK, Khaitan KB, Sethuraman G, Sharma VK (2008) Betamethasone oral mini pulse therapy compared with topical triamcinolone acetonide $(0.1 \%)$ paste in oral lichen planus: A randomized comparative study. J Am Acad Dermatol 58: 596-602. 
Citation: Dupare A, Dhole AS, Motwani M (2018) Oral Mini Pulse Therapy a Novel Therapy in a Management of Multivariant Oral Lichen Planus: An Unusual Case Report. Oral health case Rep 4: 145. doi:10.4172/2471-8726.1000145

Page 4 of 4

11. Mittal R, Manchanda $Y$ (2000) Lichen planus treated with betamethasone oral mini pulse therapy. Indian J Dermatol Venereol Leprol 66: 34-35.

12. Joshi A, Khaitan KB, Verma KK (1999) Generalised and bullous lichen planus treated successfully with oral mini-pulse therapy. Indian J Dermatol Venereol Leprol 65: 303-304.

13. Rashid MM, Khan AU, Sikder A, Ali E, Akhtar N (2008) Betamethasone ora mini-pulse therapy in the treatment of lichen planus. Iran J Derma 11: 99-102. 